\title{
Whole-Body Impedance Control for a Planetary Rover with Robotic Arm: Theory, Control Design, and Experimental Validation
}

\author{
Kristin Bussmann, Alexander Dietrich, and Christian Ott
}

\begin{abstract}
Future planetary rovers will gain the ability to manipulate their environment in addition to the maneuverability of current systems. For dedicated contact interaction, Cartesian impedance control is a well-established approach from numerous terrestrial applications. In this paper we will present a whole-body Cartesian impedance controller for a planetary rover equipped with a robotic arm. In contrast to classical terrestrial whole-body controllers, the issue of proper wheel force distribution will be addressed within the control framework. A global optimization solves this redundancy in the over-actuation of the mobile base while additionally handling the kinematic redundancy in the serial kinematic sub-chain of the robot. The approach is experimentally validated on the DLR Lightweight Rover Unit. It can be used for versatile manipulation in rough terrain such as encountered in planetary exploration or terrestrial search-and-rescue scenarios.
\end{abstract}

\section{INTRODUCTION}

Mobile manipulators can be employed in numerous fields of application including service robotics, planetary exploration, search-and-rescue scenarios, industrial applications, or logistics. Especially wheeled systems already play an important role in all of these areas. They are widely used, e. g. as robotic vacuum cleaners, lawn mowers, or autonomous transport vehicles. In planetary exploration, wheeled systems are predominant. While most rovers in the last decades have been designed with focus on maneuverability for exploration [1], [2], more and more new systems are equipped with versatile manipulation devices (robotic arms, hands, grippers) [3],[4], such as the DLR Lightweight Rover Unit (LRU) [5], see Fig. 1. Therefore, new control approaches are required which extend the previous objective of pure maneuverability to a combination of platform motion and dexterous manipulation, that is, whole-body control. Moreover, since the manipulation subtasks usually involve compliant interaction with the environment (e. g. sampling of soil, installation of a seismometer, etc.), established techniques such as impedance control [6] can be applied. In [7], a Cartesian impedance controller is proposed for the whole-body coordination of a robotic arm and a two-wheeled nonholonomic, mobile platform. While the authors lay the focus on the redundancy resolution via decoupled null space control, no dedicated multi-task optimization is performed.

Another way to make a wheeled mobile manipulator compliant is to control the manipulator in impedance-mode and connect the kinematically controlled mobile platform via an admittance interface [8]. Then the undercarriage follows

This work was supported by the Helmholtz Association, project alliance ROBEX, under contract number HA-304.

The authors are with the Institute of Robotics and Mechatronics, German Aerospace Center (DLR). Contact: kristin.bussmannedlr. de

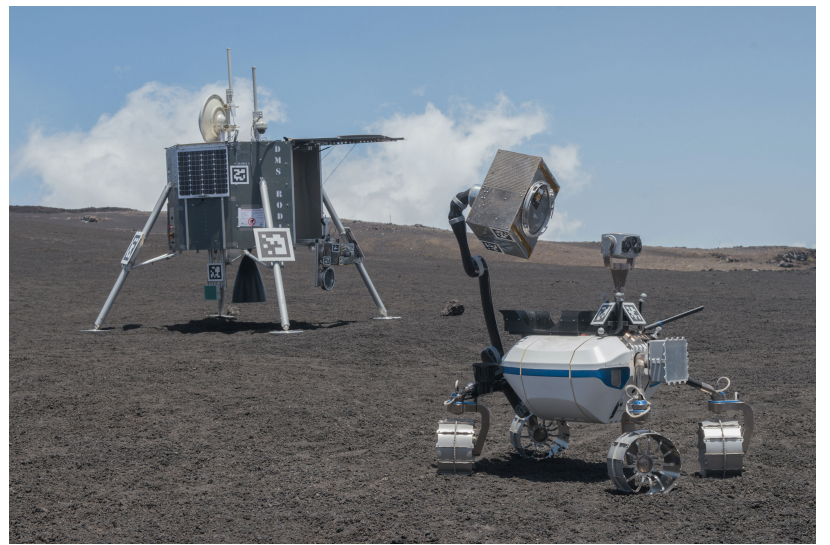

Fig. 1. The LRU manipulating a seismometer box during the ROBEX Demo Mission Space at Mt. Etna. Foto: Esther Horvath

virtual, user-defined dynamics and pretends to be forcetorque-controlled as well, reacting to external forces on the manipulator. Guaranteed stability and the parameterization of the platform inertia are strong points, but one requires an ideal kinematic controller of the base. Algorithms such as [9], [10] theoretically ensure that criterion, yet there are strong limitations in practice. But the main disadvantage is the missing compliance during physical interaction with the platform subsystem itself.

The alternative to a kinematically controlled platform is to use torque control in the wheels, if available. In case of omnidirectional wheels, one can implement mobile impedance techniques to the complete system [11], [12], [13]. In [14], a compliant whole-body controller for a wheeled humanoid robot in sloped terrains is proposed and experimentally validated. While performing well and robust in such urbanlike environments, the used omni wheels are unsuitable for planetary rovers. Unidirectional wheels, as usually used in rovers, however have to be treated differently due to the nonholonomic constraints [15]. The controller requires access to the torque in propulsion direction, and the wheels have to be aligned consistently w. r. t. the instantaneous center of rotation.

The question of how to distribute the forces and torques in wheeled undercarriages is a classical topic in platform motion control. While being a special case of the general research field of control allocation, it has not yet been investigated in the context of integrated whole-body compliance control. In [16], the authors proposed a wheel torque controller to limit slip and improve the climbing capabilities for the six-wheeled Shrimp rover. The control of a similar system, the six-wheeled CRAB rover, has been addressed in 
[17] involving the comparison with a standard velocity controller. In [18] two torque distributions have been developed and simulated on a caster-wheel-based platform. The authors were able to avoid slip and the violation of actuator limits. In [19], the wheel torques have been distributed to improve traction and reduce the power consumption, depending on the local terrain profile.

In this paper we present an impedance-based whole-body controller for a planetary rover which is equipped with a torque-controlled robotic arm. Since the control goal is to realize a Cartesian impedance behavior at the end-effector, the kinematic redundancy of the serial kinematic subchain has to be dealt with by imposing a task hierarchy. Moreover, a second kind of redundancy is inevitably introduced due to the force distribution problem in the four-wheeled mobile base. Lastly, unilateral constraints such as joint torque limits have to be incorporated. We address all these aspects simultaneously within one single optimization problem. The proposed approach is experimentally validated on the LRU (Fig. 1) in the two major aspects: the wheel force distribution and the Cartesian whole-body impedance control performance. To the best of our knowledge, this is the first controller for a rover which is capable of dealing with these aspects simultaneously within one single framework (compliant Cartesian behavior at the end-effector, proper wheel force distribution, task prioritization, unilateral constraints).

The paper is organized as follows: In Sec. II, the mathematical model of the rover is described and the control approach is introduced. Experimental results are presented in Sec. III, and the discussion follows in Sec. IV.

\section{SyStem MODEL AND CONTROL APPROACH}

Fig. 2 shows the sketch of a mobile manipulator consisting of a robotic arm mounted on a platform with steering wheels. The system model and the compliant control approach will be described in the following.

\section{A. Modeling of the mobile manipulator}

The system coordinates $\boldsymbol{z}^{T}=\left(\begin{array}{ll}\boldsymbol{\xi}^{T} & \boldsymbol{\eta}^{T}\end{array}\right) \in \mathbb{R}^{n_{\xi}+n_{\mathrm{m}}}$ are composed of the unconstrained degrees of freedom of the mobile platform, here chosen on a planar surface as $\boldsymbol{\xi}=\left(\begin{array}{lll}{ }^{0} x & { }^{0} y & \theta\end{array}\right)^{T}, n_{\xi}=3$, and the joint coordinates of the manipulator $\boldsymbol{\eta} \in \mathbb{R}^{n_{\mathrm{m}}}$. Additionally, the platform features $n_{\mathrm{w}}$ independent, centered steering wheels, and $\phi \in \mathbb{R}^{n_{\mathrm{w}}}$ is the vector of steering angles. Neglecting the steering dynamics, the equations of motion of the system are

$$
\begin{aligned}
\bar{M}(\boldsymbol{z}) \ddot{z}+\overline{\boldsymbol{C}}(\boldsymbol{z}, \dot{\boldsymbol{z}}) \dot{\boldsymbol{z}}+\overline{\boldsymbol{g}}(\boldsymbol{z}) & =\boldsymbol{\tau}+\boldsymbol{\tau}_{\mathrm{ext}}+\boldsymbol{A}(\boldsymbol{z}, \phi)^{T} \boldsymbol{\lambda} \\
\boldsymbol{A}(\boldsymbol{z}, \phi) \dot{\boldsymbol{z}} & =\mathbf{0}
\end{aligned}
$$

where $\bar{M}(\boldsymbol{z}) \in \mathbb{R}^{\bar{n} \times \bar{n}}, \bar{n}=n_{\xi}+n_{\mathrm{m}}$ is the symmetric, positive definite inertia matrix, $\overline{\boldsymbol{C}}(\boldsymbol{z}, \dot{\boldsymbol{z}}) \dot{\boldsymbol{z}} \in \mathbb{R}^{\bar{n}}$ contains centrifugal/Coriolis terms, and $\bar{g} \in \mathbb{R}^{\bar{n}}$ accounts for gravitational effects. The generalized forces acting on the system due to actuation are denoted by $\tau \in \mathbb{R}^{\bar{n}}$. In general, they can be written as

$$
\boldsymbol{\tau}=\bar{B}(\boldsymbol{z}, \phi) \boldsymbol{u},
$$

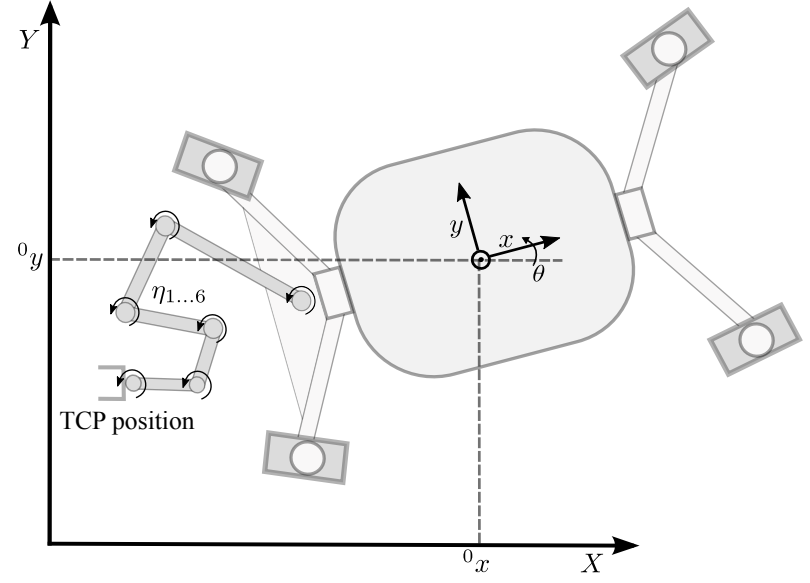

Fig. 2. Schematic drawing of the LRU. The platform has $n_{\mathrm{w}}=4$ steering wheels and the manipulator has $n_{\mathrm{m}}=6$ rotational joints.

where $\boldsymbol{u} \in \mathbb{R}^{m}$ are the $m$ actuator forces and $\overline{\boldsymbol{B}}(\boldsymbol{z}, \boldsymbol{\phi}) \in \mathbb{R}^{\bar{n} \times m}$ maps the actuator forces to generalized forces acting in $z$. Additionally, generalized external forces $\tau_{\text {ext }} \in \mathbb{R}^{\bar{n}}$ may be present.

The linear velocity constraints (2) with constraint Jacobian matrix $\boldsymbol{A}(\boldsymbol{z}, \phi) \in \mathbb{R}^{k \times \bar{n}}$ are induced by the rollingwithout-slipping conditions of the wheels. Thus, $k$ equals the number of wheels in general. The rank of the constraint Jacobian matrix $r=\operatorname{rank}(\boldsymbol{A}) \leq k$ indicates the number of linear independent constraints. For modeling, it is beneficial to reduce $\boldsymbol{A}$ to a subset of linear independent constraints such that $k=r$. The constraints are non-integrable and thus nonholonomic. The corresponding constraint forces in (1) can be written as $\boldsymbol{A}(\boldsymbol{z}, \boldsymbol{\phi})^{T} \boldsymbol{\lambda}$, which can be easily verified by the principle of virtual work. The Lagrangian multipliers $\boldsymbol{\lambda} \in \mathbb{R}^{k}$ can be interpreted as reaction forces orthogonal to feasible motion directions.

The constraint forces can be eliminated by a coordinate transformation

$$
\dot{z}=S(z, \phi) v
$$

such that $\boldsymbol{A}(\boldsymbol{z}, \phi) \boldsymbol{S}(\boldsymbol{z}, \boldsymbol{\phi})=\mathbf{0}$ with a full-rank matrix $\boldsymbol{S}(\boldsymbol{z}, \boldsymbol{\phi}) \in \mathbb{R}^{\bar{n} \times n}, n=\bar{n}-r$. This transformation describes the $n$ velocities $\boldsymbol{v} \in \mathbb{R}^{n}$ that are instantaneously feasible w.r.t. the nonholonomic constraints. The dynamics (1) can then be transformed to ${ }^{1}$

$$
\boldsymbol{M} \dot{\boldsymbol{v}}+\boldsymbol{C} \boldsymbol{v}+\boldsymbol{g}=\boldsymbol{B} \boldsymbol{u}+\boldsymbol{S}^{T} \boldsymbol{\tau}_{\mathrm{ext}}
$$

with

$$
\begin{aligned}
\boldsymbol{M} & =\boldsymbol{S}^{T} \overline{\boldsymbol{M}} \boldsymbol{S}, \\
\boldsymbol{C} & =\boldsymbol{S}^{T} \overline{\boldsymbol{M}} \dot{\boldsymbol{S}}+\boldsymbol{S}^{T} \overline{\boldsymbol{C}} \boldsymbol{S}, \\
\boldsymbol{g} & =\boldsymbol{S}^{T} \overline{\boldsymbol{g}}, \\
\boldsymbol{B} & =\boldsymbol{S}^{T} \overline{\boldsymbol{B}} .
\end{aligned}
$$

From the above equations, it becomes clear that the configuration of the steering angles influences the feasible motion directions of the platform. At the LRU, a kinematic platform

\footnotetext{
${ }^{1}$ Dependencies on state variables will be omitted in the following notations for the sake of brevity.
} 
controller makes sure that the wheels are oriented and aligned according to a desired motion direction. Thereby, desired steering angles are generated by underlying position controllers, which are not described in detail here. When operating in rough terrain (e.g. sandy underground), steering can lead to problems concerning the wheel-ground interaction, such as twisting or side-slipping of the whole rover. Thus, an arbitrary but static configuration of the steering angles is assumed for impedance-based manipulation tasks assigned to the rover. Particularly, this implies that (5) becomes a holonomic equation, with the instantaneously appointed platform motion direction as holonomic coordinate.

\section{B. Cartesian impedance control}

The transformation between joint-space velocities and Cartesian end-effector velocities can be written as

$$
\dot{\boldsymbol{x}}=\boldsymbol{J} \boldsymbol{v},
$$

with the Jacobian matrix $\boldsymbol{J} \in \mathbb{R}^{6 \times n}$. Conversely, a Cartesian force $\boldsymbol{F}$ is mapped onto the space of feasible joint torques via

$$
\boldsymbol{S}^{T} \boldsymbol{\tau}=\boldsymbol{S}^{T} \overline{\boldsymbol{J}}^{T} \boldsymbol{F}=\boldsymbol{J}^{T} \boldsymbol{F},
$$

where $\overline{\boldsymbol{J}} \in \mathbb{R}^{6 \times \bar{n}}$ is the Jacobian matrix of the unconstrained system. To achieve physical compliance at the end-effector, Cartesian impedance control can be implemented w. r.t. the coordinates $\boldsymbol{x} \in \mathbb{R}^{6}$ of the end-effector [20]. The desired wrench $\boldsymbol{F}_{\text {imp }} \in \mathbb{R}^{6}$ can be straightforwardly derived as the gradient of a virtual spring with additional damping. Furthermore, trajectory-tracking terms can be added:

$$
\boldsymbol{F}_{\mathrm{imp}}=-\left(\frac{\partial V_{\mathrm{imp}}}{\partial \boldsymbol{x}}\right)^{T}-\boldsymbol{D} \dot{\boldsymbol{x}}+\boldsymbol{M}_{\mathrm{c}} \ddot{\boldsymbol{x}}_{\mathrm{des}}+\boldsymbol{C}_{\mathrm{c}} \dot{\boldsymbol{x}}_{\mathrm{des}}
$$

The spring potential may be defined as $V_{\mathrm{imp}}=\frac{1}{2} \tilde{\boldsymbol{x}}^{T} \boldsymbol{K} \tilde{\boldsymbol{x}}$ with the deviation $\tilde{\boldsymbol{x}}=\boldsymbol{x}-\boldsymbol{x}_{\mathrm{des}}$ from the desired values $\boldsymbol{x}_{\mathrm{des}} \in \mathbb{R}^{6}$, and the stiffness matrix $\boldsymbol{K} \in \mathbb{R}^{6 \times 6}$. Damping can be injected via the positive definite damping matrix $\boldsymbol{D} \in \mathbb{R}^{6 \times 6}$. The terms $\boldsymbol{M}_{\mathrm{c}} \ddot{\boldsymbol{x}}_{\mathrm{des}}$ and $\boldsymbol{C}_{\mathrm{c}} \dot{\boldsymbol{x}}_{\mathrm{des}}$ with the taskspace inertia- and Coriolis/centrifugal matrices

$$
\begin{aligned}
\boldsymbol{M}_{\mathrm{c}} & =\left(\boldsymbol{J}^{\boldsymbol{M +}}\right)^{T} \boldsymbol{M} \boldsymbol{J}^{\boldsymbol{M +}}, \\
\boldsymbol{C}_{\mathrm{c}} & =\left(\boldsymbol{J}^{\boldsymbol{M +}}\right)^{T} \boldsymbol{C} \boldsymbol{J}^{\boldsymbol{M +}}+\left(\boldsymbol{J}^{\boldsymbol{M +}}\right)^{T} \boldsymbol{M} \dot{\boldsymbol{J}}
\end{aligned}
$$

are feed-forward terms to improve trajectory tracking, which can be omitted without affecting the contact compliance properties of the controlled system. Thereby, $\boldsymbol{J}^{M+}$ describes the dynamically consistent pseudoinverse of $\boldsymbol{J}$, see [21]. Gravity is assumed to be compensated on joint level. Passivity and stability of (5) with (12) can be shown ${ }^{2}$.

For kinematically redundant manipulators w.r.t. the Cartesian coordinates of the end-effector, i. e. $n>6$, there remains a null space in which the robot can reconfigure without affecting $\boldsymbol{x}$. In this case, additional tasks can be implemented in the null space of the main task, as shown in [22], for example. One possibility is to resolve this redundancy via numerical optimization.

\footnotetext{
${ }^{2}$ under the assumption of a static configuration of the steering angles, that is, (5) equals a holonomic system.
}

\section{Formulation of the optimization-based whole-body} impedance controller

The wrench created by the impedance controller (12) can be implemented by suitable actuator torques. Thereby, different kinds of redundancies can be observed at the system described in Sec. II-A, namely kinematic redundancy and over-actuation of the wheeled subsystem in its feasible motion directions. Thus, the choice of actuator torques is not unambiguous. An elegant solution to this problem is to apply numerical optimization strategies. One benefit is the possibility to include additional constraints such as unilateral torque limits or permissible joint angle ranges. Thus, the input variables might be chosen by minimizing the following quadratic cost function:

$$
\begin{aligned}
J & =J_{u}+J_{\mathcal{R}}+J_{\mathcal{N}} \\
J_{u} & =\frac{1}{2} \boldsymbol{u}^{T} \boldsymbol{Q}_{u} \boldsymbol{u} \\
J_{\mathcal{R}} & =\frac{1}{2}\left(\boldsymbol{F}_{\mathrm{imp}}-\boldsymbol{F}\right)^{T} \boldsymbol{Q}_{\mathcal{R}}\left(\boldsymbol{F}_{\mathrm{imp}}-\boldsymbol{F}\right), \\
J_{\mathcal{N}} & =\frac{1}{2}\left(\boldsymbol{N} \boldsymbol{\tau}_{\mathcal{N}, \text { des }}-\boldsymbol{\tau}_{\mathcal{N}}\right)^{T} \boldsymbol{Q}_{\mathcal{N}}\left(\boldsymbol{N} \boldsymbol{\tau}_{\mathcal{N}, \text { des }}-\boldsymbol{\tau}_{\mathcal{N}}\right)
\end{aligned}
$$

The input-force-related component (16) is responsible for the distribution of the inputs $\boldsymbol{u}$ w.r.t. the weighting matrix $\boldsymbol{Q}_{u} \in \mathbb{R}^{m \times m}$. It is used to solve the control allocation problem of the wheeled subsystem. The wrench-related component (17) ensures that the desired end-effector impedance is realized w.r.t. the weighting matrix $Q_{\mathcal{R}} \in \mathbb{R}^{6 \times 6}$. A secondary task can be added, e.g. on joint level, via the torque-related component (18) w.r.t. the weighting matrix $\boldsymbol{Q}_{\mathcal{N}} \in \mathbb{R}^{n \times n}$. The associated torque $\boldsymbol{\tau}_{\mathcal{N}}$ is chosen in the null space of the task Jacobian matrix ${ }^{3}$, i. e.

$$
\tau_{\mathcal{N}}=N B u
$$

The null space projector can be computed via

$$
\begin{aligned}
\boldsymbol{N} & =\boldsymbol{I}-\boldsymbol{J}^{T}\left(\boldsymbol{J}^{T}\right)^{\boldsymbol{W}}, \\
\boldsymbol{J}^{\boldsymbol{W}+} & =\boldsymbol{W}^{-1} \boldsymbol{J}^{T}\left(\boldsymbol{J} \boldsymbol{W}^{-1} \boldsymbol{J}^{T}\right)^{-1} .
\end{aligned}
$$

wherein $\boldsymbol{J}^{\boldsymbol{W}+}$ describes the weighted pseudoinverse w.r.t. the weighting matrix $\boldsymbol{W} \in \mathbb{R}^{n \times n}$. If $\boldsymbol{W}=\boldsymbol{M}$, the projection is dynamically consistent [21]. That is, $\boldsymbol{\tau}_{\mathcal{N}}$ does not induce accelerations in the Cartesian space of the endeffector. To make sure that the vertex of the cost function $\boldsymbol{J}_{\mathcal{N}}$ can be reached during the optimization, the desired torque $\tau_{\mathcal{N} \text {,des }}$ needs to be projected into the null space as well (cf. (18)).

The global optimization problem can then be established as:

$$
\begin{array}{ll}
\qquad \boldsymbol{u}^{*}=\arg \min J \\
\text { s.t. } & (\boldsymbol{I}-\boldsymbol{N}) \boldsymbol{B} \boldsymbol{u}=\boldsymbol{J}^{T} \boldsymbol{F} \\
& \boldsymbol{N} \boldsymbol{B} \boldsymbol{u}=\boldsymbol{\tau}_{\mathcal{N}} \\
& \boldsymbol{u}_{\min }<\boldsymbol{u}<\boldsymbol{u}_{\max }
\end{array}
$$

\footnotetext{
${ }^{3}$ The indices $\mathcal{R}$ and $\mathcal{N}$ indicate range space and null space of the task Jacobian matrix, respectively.
} 
with the permissible lower and upper bounds $\boldsymbol{u}_{\min }$ and $\boldsymbol{u}_{\text {max }}$, respectively. The constraints (23) and (24) are used to implement (17) and (18) w.r.t. the inputs $\boldsymbol{u}$.

Note that this specific formulation of the control law as a quadratic optimization problem with linear constraints facilitates fast online computation of the solution. The side conditions can be extended arbitrarily according to the specifications of the system. Futhermore, if unilateral constraints are active, the sum of null space and range space torques is optimized according to the weight of the task, which leads to a different behavior of the controlled system compared to strict null space projection approaches. This will be discussed w. r. t. experimental results in Sec. III-B.

\section{EXPERIMENTS}

The experiments on the mobile base are performed on the LRU introduced in Sec. I. The rover was designed as a research platform for planetary exploration and terrestrial search-and-rescue scenarios. To guarantee mobility in rough terrain, the rover is equipped with four individually steerable wheels that provide both a velocity and a torque control input in spinning direction. A 6-degree-of-freedom robotic arm (JACO, built by Kinova) is mounted on the rover for manipulation purposes. The optimization problems were solved using qpOASES, an open-source implementation of an active-set method for quadratic programming [23].

In the first part of the experiments, the focus lies on examining the distribution of the wheel forces. Therefore, an impedance control law is applied only onto the mobile platform. In the second part of this section, experiments with the complete mobile manipulator were performed, including end-effector positioning tasks, but also contact situations. This part of the experiments is also shown in the video that accompanies this paper. Note that in the following experiments, the tracking terms were omitted in the Cartesian impedance control law (12).

\section{A. Distribution of wheel forces}

In the following, the impedance control law is exclusively applied to the platform. A trajectory in $x$-direction is commanded for the center of the rover and the resulting impedance force is generated by the wheels. The corresponding optimization problem was implemented as a reduced version of (22)-(25):

$$
\begin{aligned}
\boldsymbol{f}^{*}= & \arg \min \frac{1}{2} \boldsymbol{f}^{T} \tilde{\boldsymbol{Q}}_{u} \boldsymbol{f}+\frac{1}{2} \boldsymbol{s}^{T} \tilde{\boldsymbol{Q}}_{\mathcal{R}} \boldsymbol{s} \\
\text { s.t. } & \tilde{\boldsymbol{B}} \boldsymbol{f}-\tilde{\boldsymbol{F}}_{\mathrm{imp}}=\boldsymbol{s} . \\
& \boldsymbol{f}_{\min }<\boldsymbol{f}<\boldsymbol{f}_{\max }
\end{aligned}
$$

where $f \in \mathbb{R}^{4}$ are the actuator forces commanded to the wheels, $\tilde{\boldsymbol{F}}_{\text {imp }} \in \mathbb{R}^{3}$ is the impedance wrench acting in the plane, $s \in \mathbb{R}^{3}$ is the error between impedance force and the force generated by the optimization, and $\tilde{\boldsymbol{Q}}_{u} \in \mathbb{R}^{4 \times 4}$ and $\tilde{Q}_{\mathcal{R}} \in \mathbb{R}^{3 \times 3}$ are the corresponding weighting matrices.

In the first experiment, the wheel torque distribution minimizing the wheel forces is compared to a wheel torque distribution minimizing the wheel powers. For this purpose,
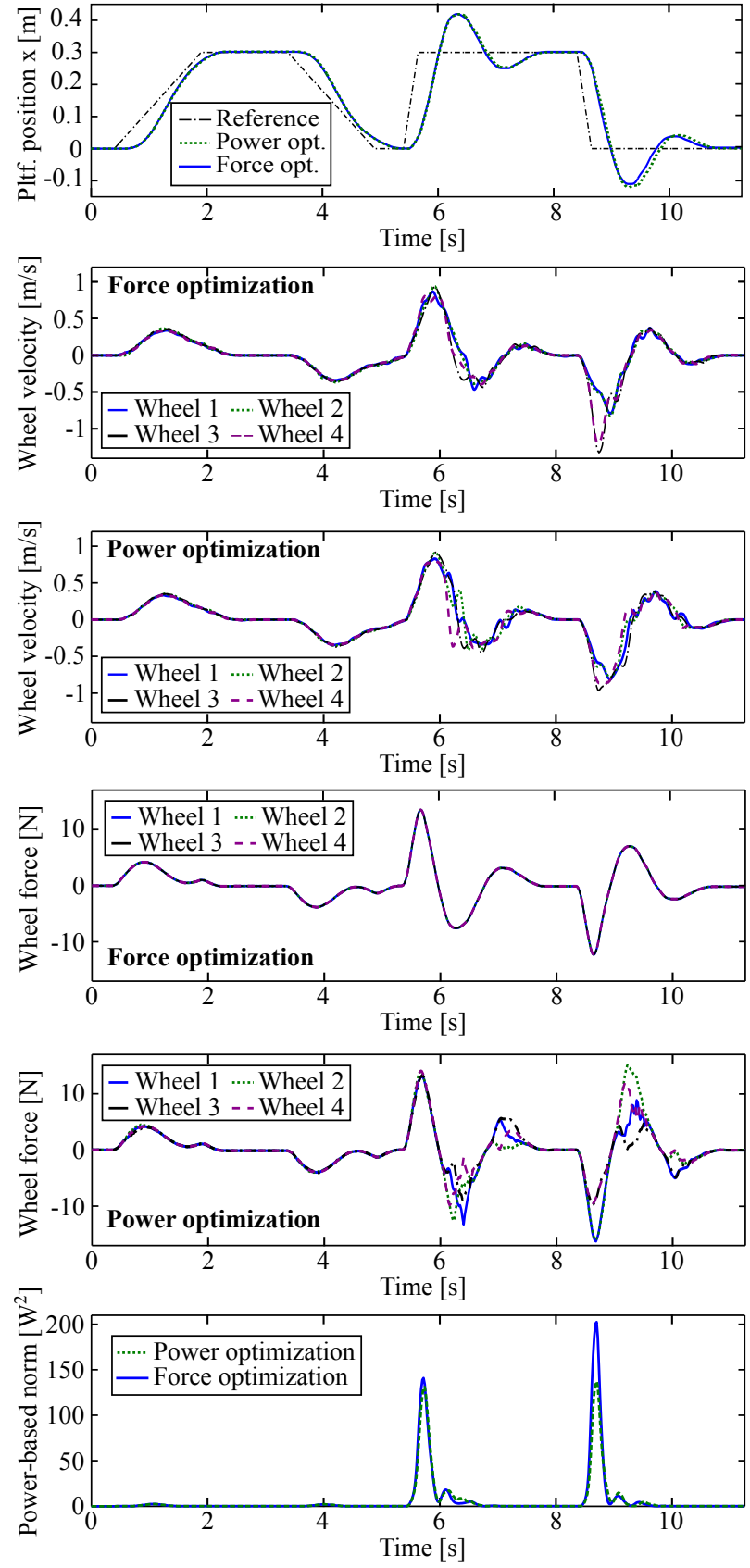

Fig. 3. Top plot: Reference trajectory and system response. Diagrams 25: Wheel velocity measurements and corresponding optimized wheel force commands. Bottom plot: comparison of the power-based norm for the two optimization approaches.

the weighting matrix $\tilde{\boldsymbol{Q}}_{u}$ in (26) is chosen as $\tilde{\boldsymbol{Q}}_{u}^{I}=\boldsymbol{I}_{4 \times 4}$ (the $4 \times 4$ unit matrix) or $\tilde{\boldsymbol{Q}}_{u}^{v}=\operatorname{diag}\left(v_{\mathrm{w}, 1}^{2}, v_{\mathrm{w}, 2}^{2}, v_{\mathrm{w}, 3}^{2}, v_{\mathrm{w}, 4}^{2}\right)$, respectively. Thereby, the measurements of the wheel velocities are denoted as $v_{\mathrm{w}, i}$ for $i=1 \ldots 4$. Throughout the experiment, the rear wheels are located on a surface with different friction properties than the front wheels. This setup is inspired by planetary exploration scenarios, where the rover must be able to cope with locally different soil properties. In Fig. 3 (top plot), the reference trajectory and the resultant platform motion in $x$-direction are shown for the two cases of force and power optimization. The second and the third plot depict the wheel velocity measurements, the two plots below 

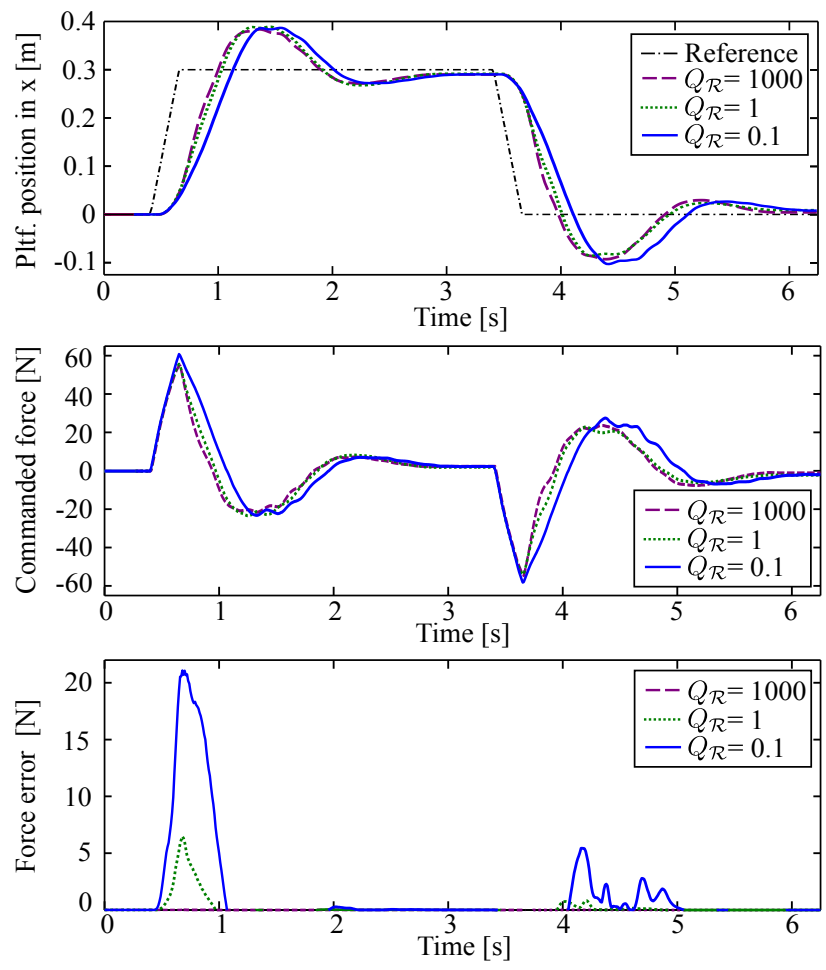

Fig. 4. Top plot: reference trajectory and resulting platform motion for different parameterizations of the weighting factor $Q_{\mathcal{R}}\left[\frac{\mathrm{m}^{2}}{\mathrm{~s}^{2}}\right]$ for the force error $s$. Bottom plots: commanded force $F_{x, \text { cmd }}$ and force error $F_{x, \text { cmd }}-F_{x, \text { res. }}$. The resulting force $F_{x, \text { res }}$ is the sum of all wheel forces.

show the corresponding wheel force commands generated in the two different force optimization approaches. During the first part of the trajectory $(0 \mathrm{~s}<t<5 \mathrm{~s})$, the trapezoid reference signal has a small slope and thus produces moderate commanded forces that do not lead to wheel slippage. Therefore, the two optimization procedures generate almost identical wheel force commands. The second part of the reference trajectory $(5 \mathrm{~s}<t<10 \mathrm{~s})$ is characterized by a large slope and yields a high amplitude in the commanded force. In the velocity measurements it can be seen that wheels 3 and 4 lose traction and thus start to spin during the fast backward motion at $8 \mathrm{~s}<t<10 \mathrm{~s}$. In the case of force optimization, the velocity measurements are not used in the computation of the wheel forces. Therefore the wheel velocities get large, especially at $t \approx 9 \mathrm{~s}$. Only the decrease in the commanded force leads to a reduction of the spinning velocities. In the case of power optimization, it can be seen that the forces of wheel 3 and 4 are reduced after an initial increase of the corresponding velocities. The force is redistributed to wheels 1 and 2 such that the resulting force in $x$-direction is retained. The effect on the overall power consumption of the rover can be inspected in the bottom plot of Fig. 3, where the (squared) power-based norm $\|\boldsymbol{f}\|_{v}^{2}=\frac{1}{2} \boldsymbol{f}^{T} \tilde{\boldsymbol{Q}}_{u}^{v} \boldsymbol{f}$ is plotted. The value of the power norm is reduced significantly in the case of slippage of two out of four wheels.

In the second experiment, a trajectory with steep ascent of the desired position is commanded in order to induce a high force that causes slip in all wheels. The reference position
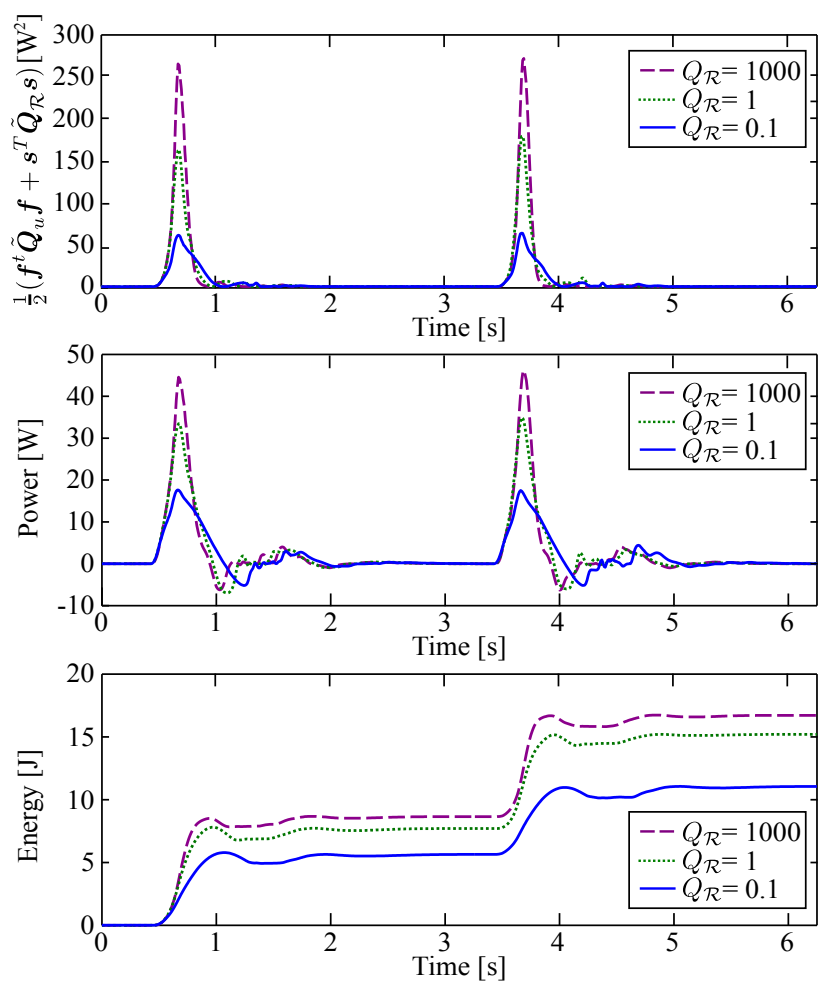

Fig. 5. Power-based norm, power and energy for different values of the force error variable weighting factor $Q_{\mathcal{R}}\left[\frac{\mathrm{m}^{2}}{\mathrm{~s}^{2}}\right]$

and the resulting platform motion are depicted in the top plot of Fig. 4. The next plot shows the resulting commanded forces produced by the high-level controller. In the bottom plot of Fig. 4, the force error $F_{\mathrm{imp}, x}-F_{x}$ is depicted for different values of $Q_{\mathcal{R}}$ (the weighting factor of the force error in $x$-direction). Here, the resulting force $F_{x \text {,res }}$ is the sum of all wheel forces obtained by a power optimization according to (26). In Fig. 5, the squared power-based norm $\|\boldsymbol{f}\|_{v}^{2}=\frac{1}{2} \boldsymbol{f}^{T} \tilde{\boldsymbol{Q}}_{u}^{v} \boldsymbol{f}+\frac{1}{2} \boldsymbol{s}^{T} \tilde{\boldsymbol{Q}}_{\mathcal{R}} \boldsymbol{s}$, the power $P=\sum_{i=1}^{4} F_{i} v_{i}$, and the energy $E=\int_{0}^{t} P \mathrm{~d} \tilde{t}$ are plotted. For $Q_{\mathcal{R}}=1000 \frac{\mathrm{m}^{2}}{\mathrm{~s}^{2}}$, the resulting force is very close to the commanded force. For $Q_{\mathcal{R}}=1 \frac{\mathrm{m}^{2}}{\mathrm{~s}^{2}}$, the values of the weighting factors for the power and for the deviation of the resultant force are in the same range (cf. Fig. 3). The error between commanded and resultant force in Fig. 4 (bottom plot, green dashed line) is small compared to the total value of the commanded force. Also the resulting platform motion is similar to the case when $Q_{\mathcal{R}}=1000 \frac{\mathrm{m}^{2}}{\mathrm{~s}^{2}}$. Nevertheless, the force deviation leads to reduced wheel slippage and thus to a significant difference in the power consumption, which becomes visible in Fig. 5. Comparing the energies in the bottom plot it becomes clear that approximately $1 \mathrm{~J}$ is saved each time a rapid movement occurs. Thinking of limited resources (e.g. in planetary exploration missions where battery-based power supply is limited), the importance of such savings related to marginal losses in performance becomes evident. In the case of $Q_{\mathcal{R}}=0.1 \frac{\mathrm{m}^{2}}{\mathrm{~s}^{2}}$, the generated force deviates from the desired one with up to $20 \mathrm{~N}$, which leads to a noticeably slower system response, but also to energy savings up to $4 \mathrm{~J}$ per acceleration process compared to the case when 

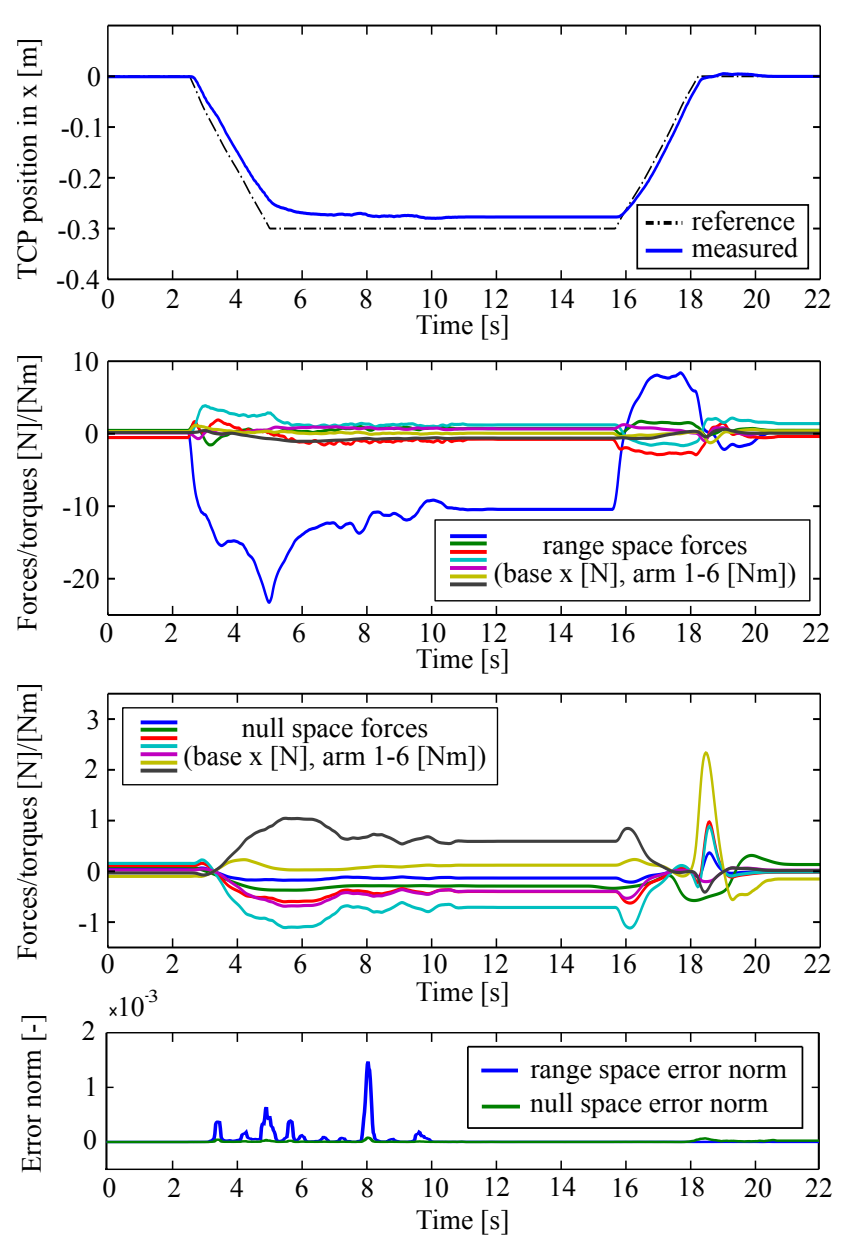

Fig. 6. Top plot: commanded and measured $x$-coordinate of the TCP. Second plot: commanded joint torques in the range space of the transposed Jacobian matrix, created by optimization of the end-effector wrench. Blue line: Platform force in $x$-direction created by all wheels. Other lines: Joint torques $1 . .6$ of the robotic arm. Third plot: commanded joint torques in the null space of the task Jacobian matrix. Bottom plot: Normed force/torque errors in range space and null space

$Q_{\mathcal{R}}=1000 \frac{\mathrm{m}^{2}}{\mathrm{~s}^{2}}$, see Fig. 5 (bottom diagram). Note that the goal position is still reached despite the force deviation, as the superimposed controller compensates for errors in the underlying torque controller.

\section{B. Whole-body impedance control}

In the second part of the experiments, the proposed Cartesian impedance controller is implemented according to (22)-(25) on the complete system, i.e. the wheeled platform and the 6-degree-of-freedom robotic arm. The desired Cartesian stiffness was set to $450 \frac{\mathrm{N}}{\mathrm{m}}$ for translations and $12.5 \frac{\mathrm{Nm}}{\mathrm{rad}}$ for rotations, all damping ratios were chosen as 0.5. The wheels were oriented such that only platform motions in $x$-direction were possible. The null space task was a joint impedance with stiffness $20 \frac{\mathrm{Nm}}{\mathrm{rad}}$ in all arm joints, the desired values were set close to the initial arm configuration. No damping was added in the null space, since the JACO arm features large intrinsic joint damping. For the optimization, the following gains were chosen ${ }^{4}$ :

\footnotetext{
${ }^{4}$ The units in the weighting matrices are chosen consistently such that $J$ is unit-free.
}
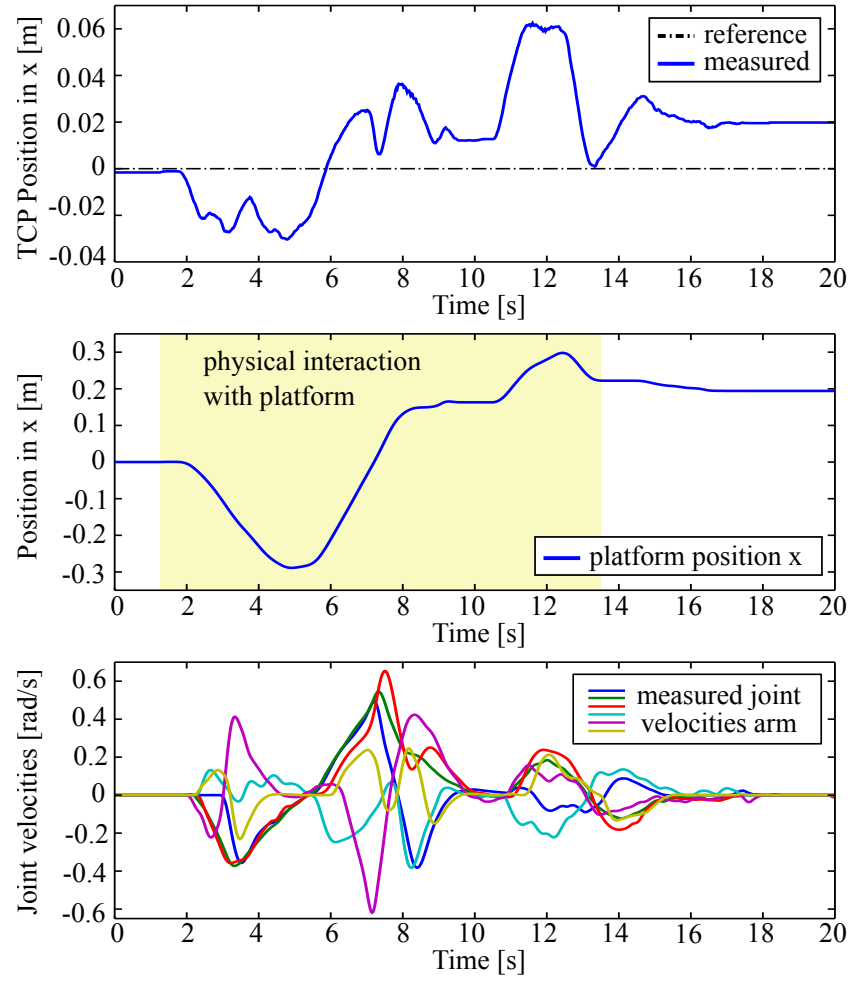

Fig. 7. Physical interaction with the platform. Top plot: commanded and measured $x$-coordinate of the TCP. Second plot: position of the platform in $x$-direction. Third plot: measured joint velocities in the robotic arm

$\boldsymbol{Q}_{u}=\operatorname{diag}\left(v_{\mathrm{w}, 1}^{2}, v_{\mathrm{w}, 2}^{2}, v_{\mathrm{w}, 3}^{2}, v_{\mathrm{w}, 4}^{2}, 0,0,0,0,0,0\right), \boldsymbol{Q}_{\mathcal{R}}=$ $\operatorname{diag}(1,1,1,1,1,1), \boldsymbol{Q}_{\mathcal{N}}=\operatorname{diag}(0,1,1,1,1,1,1)$.

At first the regulation of a desired end-effector position and orientation was evaluated. The uppermost plot in Fig. 6 shows the transient of the TCP in $x$-direction. The soft spring yields a steady-state error, which is natural for impedance control and, in this case, mainly due to unmodeled friction between wheels and underground. That can be seen in the second plot, where the range space torques $\boldsymbol{\tau}_{\mathcal{R}}=\boldsymbol{B} \boldsymbol{u}_{\mathcal{R}}$ are plotted. From approx. $11 \mathrm{~s}$ to $16 \mathrm{~s}$, the commanded forces are constant as the robot does not move. The blue line shows the relatively high force in platform $x$-direction, which does not induce a movement of the platform, which leads to a static error at the end-effector. The third plot shows the null space torques, which keep the joints close to their initial configuration without affecting the end-effector wrench. Note that without a dedicated null space reference torque, the steadystate error in the end-effector position could be compensated by the arm and thus be significantly smaller. As no actuator torque limits are met, both range space force and null space torque could be accomplished without limitations. This can be verified in the bottom plot, where the error norm of the end-effector wrench, $\sqrt{J_{\mathcal{R}}}$ (blue), and the null space error norm $\sqrt{J_{\mathcal{N}}}$ (green) are depicted.

Now we investigate and highlight the differences between the presented whole-body controller and approaches such as [8], in which the platform controller is kinematic and the overall impedance behavior is generated via an admittance interface. Within the whole-body control framework at hand, the platform is controlled on torque level and thus shows 

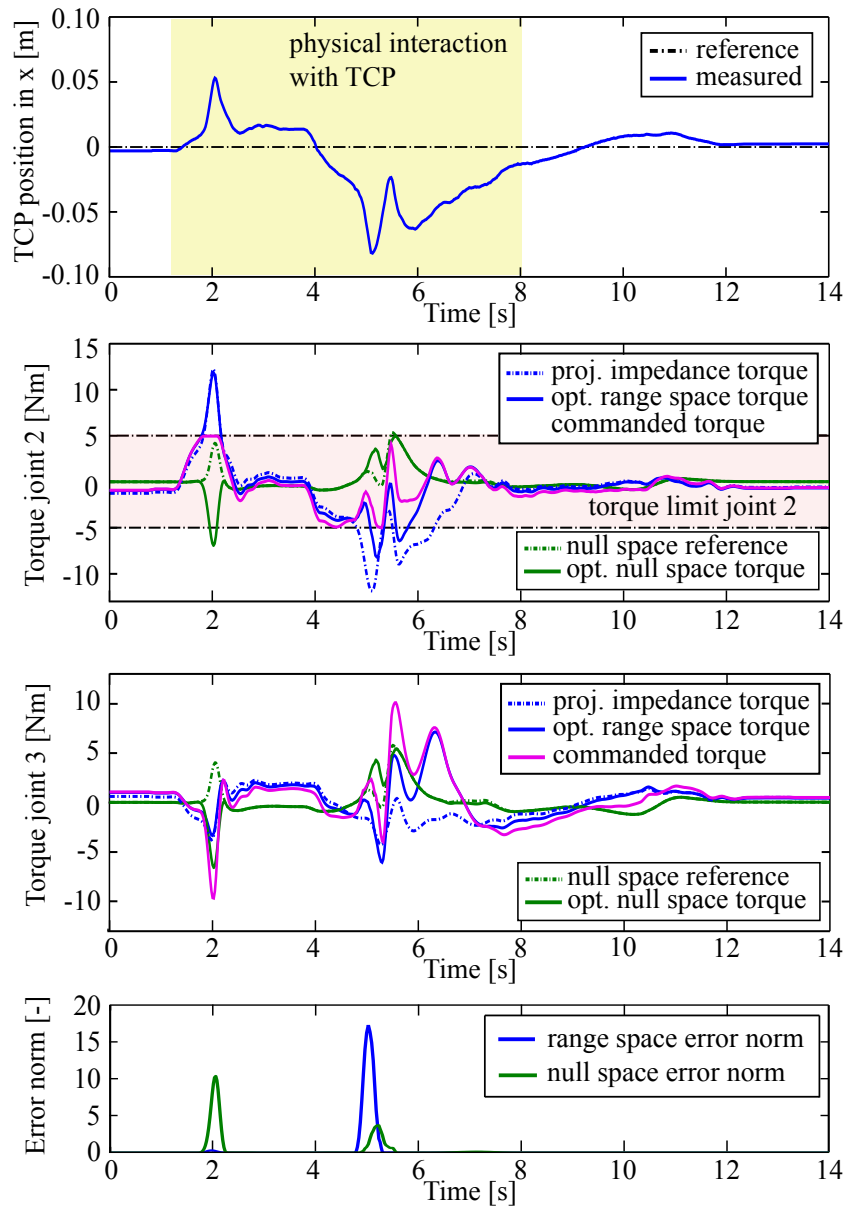

Fig. 8. Physical interaction at the TCP in the presence of joint torque limits. Top plot: commanded and measured $x$-coordinate of the TCP. Second plot: torques generated at the second joint (constrained to $5 \mathrm{Nm}$ ). Third plot: torques generated at the third joint (unconstrained). Bottom plot: Norm of the force/torque errors in range space and null space

actively compliant behavior. In Fig. 7, it is shown how a user actively moves the wheeled subsystem in the null space of the end-effector. This is only possible when the wheels are back-drivable. Note that no explicit platform positioning subtask is implemented in the null space. The second plot shows the $x$-coordinate of the platform, which is pushed and pulled such that the overall position changes about $0.5 \mathrm{~m}$. Thereby, the end-effector position is approximately constant, as can be verified in the first plot. During the physical interaction with the platform, the joints of the arm move in order to keep the TCP position at its desired value. The velocities measured in the arm joints indicate that in the bottom plot. In general, active compliance of the locomotion subsystem of a wheeled manipulator can be beneficial both in autonomous manipulation (e.g. in the presence of obstacles) and in human-robot interaction scenarios, where compliant behavior can be safety-critical. That is a major advantage of the presented approach compared to [8].

Finally, the behavior in the presence of constraints was examined. Therefore, the diagonal entries of $\boldsymbol{Q}_{\mathcal{N}}$ were set to 0.1 , such that the main task was weighted ten times higher than the null space task. The joint torque of joint 2 (elbow joint of the arm) was limited to $5 \mathrm{Nm}$ ( $18 \mathrm{Nm}$ peak torque in data sheet). The constrained behavior was tested during physical interaction at the end-effector. The interaction period is highlighted in the upper plot in Fig. 8, which shows the induced deflection of the $x$-coordinate of the end-effector. The interaction can be roughly divided into two phases: In phase 1 from about $1 \mathrm{~s}$ to $4 \mathrm{~s}$, a user pushes the TCP (positive error). Afterwards, the user pulls the robot, which leads to a negative error. The second plot shows the resulting torques in the constrained second joint, the range of admissible torques is marked. The projected impedance torque (blue, dot-dashed line) would be generated by a classical impedance controller without optimization by projection via the transposed Jacobian matrix. The green, dot-dashed line shows the null space reference torque. The full blue and green lines depict the range space and null space torques resulting from the optimization, the pink line is the superposition of both that creates the actuator command torque. In the first phase of the interaction, the impedance controller induces torques that are higher than the allowed $5 \mathrm{Nm}$. In order to keep the sum of the torques below $5 \mathrm{Nm}$, the optimization yields a deviation of the null space torque, such that the range space torque is close to its reference. Interestingly, this can be also observed in other (non-constrained) joints, which can be exemplarily examined in the third plot. There, the joint torques in joint 3 are depicted, which also show a deviation of the null space torque at the first interaction phase, even if joint 3 itself does not meet a constraint. The error norm shown in the bottom plot reveals that the controller is able to produce a large extent of the desired end-effector wrench at the cost of errors in the null space. This is a difference to strict hierarchical approaches, where constraints would not alter the null space behavior in order to achieve the desired endeffector forces. In the second phase of the interaction, the constraint w. r.t. joint 2 is active two times between $4 \mathrm{~s}$ and $6 \mathrm{~s}$. In this configuration, errors in the range space wrench cannot be fully inhibited by deviations of the null space torques, even if effects similar to the aforementioned (in the first interaction phase) can be observed at about $5 \mathrm{~s}$. Therefore, the error norm in the range space rises in this time interval. Nevertheless, the controlled system maintains stable and converges to zero after the end of the physical interaction.

\section{DISCUSSION}

The controller presented in this paper is based on the classical Cartesian impedance approach. The system characteristics of the considered rover give point to the implementation as a numerical optimization problem, but an analytical approach could also be considered in principle. A lack of analytical proofs of stability can be mentioned as one of the main disadvantages of the optimization-based approach. On the other hand, the main advantage is the consideration of unilateral constraints, which have to be taken into account in space exploration scenarios, where the systems have to operate fully autonomous. For these unilateral constraints, the classical proofs of stability for impedance-based con- 
trollers do not hold anymore. Computational load could be assumed another issue of numerical optimizations, but for the case of quadratic problems, there exist dedicated solvers that can handle the arising computations sufficiently fast, as the experiments clearly showed. Summarizing, the numerical approach features performant behavior in the presence of system limitations (as discussed in Sec. III).

As the focus of this paper was primarily the investigation of force distribution, the problem how to steer the wheels was not addressed. Due to the nature of wheeled locomotion, the rover is subject to nonholonomic constraints that restrict the feasible directions of motion of the platform subsystem. An interesting question in the context of compliance control is how to define the directions in which the compliance of the wheeled subsystem should be implemented. Possible approaches would be strongly application-dependent and range from reactive admittance-based steering parameterizations to workspace-dependent steering, to name but a few. This question will be topic of future work.

Finally, the described optimization-based whole-body impedance controller was introduced and discussed in the context of a planetary rover, but can be easily applied in other scenarios as well. Due to its generalizable formulation, it could be of use to a broad field of robotic systems, i.e. kinematically redundant mobile manipulators with torque controllable wheels. Especially the active compliance of the wheeled subsystem makes the approach suitable for manipulation scenarios involving unknown environments as well as physical human-robot interaction.

\section{CONCLUSION}

In this work, we presented an optimization-based variant of a whole-body impedance controller for a rover equipped with a torque-controlled manipulator. The system modeling and the control approach were described. Experiments with the LRU were performed, which focused on two aspects of the proposed controller: the wheel force distribution and the behavior of the whole-body controller. Finally, the approach was discussed both from a theoretical and practical viewpoint.

\section{REFERENCES}

[1] A. H. Mishkin, J. C. Morrison, T. T. Nguyen, H. W. Stone, B. K. Cooper, and B. H. Wilcox, "Experiences with operations and autonomy of the mars pathfinder microrover," in 1998 IEEE Aerospace Conference Proceedings, vol. 2, March 1998, pp. 337-351.

[2] M. Bajracharya, M. W. Maimone, and D. Helmick, "Autonomy for mars rovers: Past, present, and future," Computer, vol. 41, no. 12, pp. 44-50, Dec 2008.

[3] A. Trebi-Ollennu, E. T. Baumgartner, P. C. Leger, and R. G. Bonitz, "Robotic arm in-situ operations for the mars exploration rovers surface mission," in 2005 IEEE International Conference on Systems, Man and Cybernetics, vol. 2, Oct 2005, pp. 1799-1806.

[4] J. Schwendner, T. M. Roehr, S. Haase, M. Wirkus, M. Manz, S. Arnold, and J. Machowinski, "The Artemis rover as an example for model based engineering in space robotics," in ICRA Workshop on Modelling, Estimation, Perception and Control of All Terrain Mobile Robots, 2014.

[5] M. J. Schuster, C. Brand, S. G. Brunner, P. Lehner, J. Reill, S. Riedel, T. Bodenmüller, K. Bussmann, S. Büttner, A. Dömel et al., "The LRU Rover for Autonomous Planetary Exploration and its Success in the SpaceBotCamp Challenge," in 2016 IEEE International Conference on Autonomous Robot Systems and Competitions (ICARSC), May 2016.
[6] N. Hogan, "Impedance Control: An Approach to Manipulation: Part I - Theory, Part II - Implementation, Part III - Applications," Journal of Dynamic Systems, Measurement, and Control, vol. 107, pp. 1-24, March 1985.

[7] G. D. White, R. M. Bhatt, C. P. Tang, and V. N. Krovi, "Experimental evaluation of dynamic redundancy resolution in a nonholonomic wheeled mobile manipulator," IEEE/ASME Transactions on Mechatronics, vol. 14, no. 3, pp. 349-357, June 2009.

[8] A. Dietrich, K. Bussmann, F. Petit, P. Kotyczka, C. Ott, B. Lohmann, and A. Albu-Schäffer, "Whole-body impedance control of wheeled mobile manipulators: Stability analysis and experiments on the humanoid robot Rollin' Justin," Autonomous Robots, vol. 40, no. 3, pp. 505-517, March 2016.

[9] P. R. Giordano, M. Fuchs, A. Albu-Schäffer, and G. Hirzinger, "On the Kinematic Modeling and Control of a Mobile Platform Equipped with Steering Wheels and Movable Legs," in Proc. of the 2009 IEEE International Conference on Robotics and Automation, May 2009, pp. 4080-4087.

[10] G. Oriolo, A. D. Luca, and M. Vendittelli, "WMR control via dynamic feedback linearization: design, implementation, and experimental validation," IEEE Transactions on Control Systems Technology, vol. 10, no. 6, pp. 835-852, Nov 2002.

[11] R. Holmberg and O. Khatib, "Development and control of a holonomic mobile robot for mobile manipulation tasks," International Journal of Robotics Research, vol. 19, no. 11, pp. 1066-1074, 2000.

[12] S. Djebrani, A. Benali, and F. Abdessemed, "Modelling and control of an omnidirectional mobile manipulator," International Journal of Applied Mathematics and Computer Science, vol. 22, no. 3, pp. 601616, 2012.

[13] K. Kosuge, M. Sato, and N. Kazamura, "Mobile robot helper," in Proc. of the 2000 IEEE International Conference on Robotics and Automation, vol. 1, 2000, pp. 583-588.

[14] L. Sentis, J. Petersen, and R. Philippsen, "Implementation and stability analysis of prioritized whole-body compliant controllers on a wheeled humanoid robot in uneven terrains," Autonomous Robots, vol. 35, no. 4, pp. 301-319, 2013.

[15] G. Campion, G. Bastin, and B. D'Andréa-Novel, "Structural Properties and Classification of Kinematic and Dynamic Models of Wheeled Mobile Robots," IEEE Transactions on Robotics and Automation, vol. 12, no. 1, pp. 47-62, February 1996.

[16] P. Lamon, A. Krebs, M. Lauria, R. Siegwart, and S. Shooter, "Wheel torque control for a rough terrain rover," in Proc. of the 2004 IEEE International Conference on Robotics and Automation, vol. 5, 2004, pp. 4682-4687.

[17] A. Krebs, T. Thueer, and E. Carrasco, "Towards torque control of the crab rover," in Proc. of the 9th International Symposium on Artificial Intelligence, Robotics and Automation in Space, 2008.

[18] Y. P. Li, T. Zielinska, M. H. Ang Jr, and W. Lin, "Wheel-ground interaction modelling and torque distribution for a redundant mobile robot," in Proc. of the 2006 IEEE International Conference on Robotics and Automation, May 2006, pp. 3362-3367.

[19] K. Iagnemma and S. Dubowsky, "Traction control of wheeled robotic vehicles in rough terrain with application to planetary rovers," The International Journal of Robotics Research, vol. 23, no. 10-11, pp. 1029-1040, 2004.

[20] A. Albu-Schäffer, C. Ott, and G. Hirzinger, "A Unified Passivity-based Control Framework for Position, Torque and Impedance Control of Flexible Joint Robots," International Journal of Robotics Research, vol. 27, no. 1, pp. 23-39, January 2007.

[21] O. Khatib, "A Unified Approach for Motion and Force Control of Robot Manipulators: The Operational Space Formulation," IEEE Journal of Robotics and Automation, vol. RA-3, no. 1, pp. 43-53, February 1987.

[22] A. Dietrich, C. Ott, and A. Albu-Schäffer, "Multi-Objective Compliance Control of Redundant Manipulators: Hierarchy, Control, and Stability," in Proc. of the 2013 IEEE/RSJ International Conference on Intelligent Robots and Systems, November 2013, pp. 3043-3050.

[23] H. Ferreau, C. Kirches, A. Potschka, H. Bock, and M. Diehl, "qpOASES: A parametric active-set algorithm for quadratic programming," Mathematical Programming Computation, vol. 6, no. 4, pp. 327-363, 2014. 